\title{
Research Article \\ On Certain Products of Complex Intuitionistic Fuzzy Graphs
}

\author{
Abida Anwar (D) and Faryal Chaudhry (D) \\ Department of Mathematics, The University of Lahore, Lahore, Pakistan \\ Correspondence should be addressed to Abida Anwar; abidaaslam34@gmail.com
}

Received 27 August 2021; Revised 9 November 2021; Accepted 10 November 2021; Published 16 December 2021

Academic Editor: Sarfraz Nawaz Malik

Copyright (c) 2021 Abida Anwar and Faryal Chaudhry. This is an open access article distributed under the Creative Commons Attribution License, which permits unrestricted use, distribution, and reproduction in any medium, provided the original work is properly cited.

A complex intuitionistic fuzzy set (CIFS) can be used to model problems that have both intuitionistic uncertainty and periodicity. A diagram composed of nodes connected by lines and labeled with specific information may be used to depict a wide range of reallife and physical events. Complex intuitionistic fuzzy graphs (CIFGs) are a broader type of diagram that may be used to manipulate data. In this paper, we define the key operations direct, semistrong, strong, and modular products for complex intuitionistic fuzzy graphs and look at some interesting findings. Further, the strong complex intuitionistic fuzzy graph is defined, and several significant findings are developed. Furthermore, we study the behavior of the degree of a vertex in the modular product of two complex intuitionistic fuzzy graphs.

\section{Introduction}

Obscurity is a common occurrence in everyday life. This is not a world of precise calculations and ideas. For human intellect, this judgment mistake is extremely tough. To tackle this problem, a variety of mathematical techniques and ideas, such as fuzzy sets and complex fuzzy sets, have been developed. A group system with uncertain information was used to create the complex fuzzy logic. Due to the elastic potential of advanced intuitionistic fuzzy sets (IFSs) to control unreliability, this event is considered wonderfully great for humanistic logic underlying wrong reality and infinite knowledge. Because it allows for more erroneous information to be given, this theory is a cornerstone of classical complex fuzzy sets because it provides for more suitable answers to a range of situations. In cases when we must deal with relatively limited alternatives, such as yes or no, these specialized sets generated beneficial models. Another essential feature of this knowledge is that it allows man to evaluate the negative and positive elements of erroneous ideas. To deal with uncertainty, Zadeh [1] introduced the fuzzy set theory. Following that, a number of academics looked into the theory of fuzzy sets and fuzzy logic in order to deal with a variety of real-world problems involving an uncertain and ambiguous environment. Atanassov [2] came up with the concept of intuitionistic fuzzy sets (IFSs), which are fuzzy sets with a new component. With the addition of the degree of truth and falsity membership, the concept of IFSs has become more relevant and vivid. The applications of these sets have gotten a lot of attention in fields like multicriteria decision-making and image processing. Furthermore, when data is phase-shifted, the ambiguity and uncertainty in the data come from everyday life. As a result, taking this information into account is theoretically insufficient, and information is lost as a result of the procedure. To handle this uncertainty, Ramot et al. [3] introduced the elongated form the fuzzy set by including a phase term part, called complex fuzzy set. The competency of complex fuzzy logic in the sense of membership has a very significant role to address concrete problems. It is not only a vital source for measuring unevenness but also very effective mode to deal with ambiguous ideas. Besides its usefulness, we still have massive problems regarding the physical properties of complex membership-based functions. It is highly demanding to design an additional theory of complex fuzzy set in the sense of set knotty members. This logic is straight development of conventional fuzzy logic that naturally develops problem basing fuzzy logic which is not suitable for the artificial function of membership. This certain set has core role in various applications especially in modern commanding systems which foreshadows periodic phenomenon wherein a series of fuzzy variables are 
interlinked in a complicated way and it cannot be properly run by fuzzy operations. Owing to the feature of handling the information regarding both periodicity and uncertainty, the complex fuzzy sets gained the special attentions in the latest trends of fuzzy sets, bipolar fuzzy sets, and IFSs. By using these models, both periodicity and uncertainty may be presented in a single set. Atanassov [2] added new components to the concept of a fuzzy set that specifies the degree of nonmembership. Fuzzy sets provide membership degrees, while IFSs provide both membership and nonmembership degrees, which are more or less independent of one another. The sole requirement is that the total of the two degrees is less than one. IFSs have been used in economics, chemistry, medicine, engineering, and computer science. Therefore, the studies regarding complex fuzzy sets got a broad spectrum both in theoretical aspects as well as application aspects. Many researchers investigated the extensive applications of complex fuzzy sets in signal processing applications, time series, solar activity, and forecasting problems (for instance, see [4-8]). Complex numbers and complex fuzzy sets were utilized by Buckley [9]. Alkouri and Salleh [10] developed the concept of complex Atanassov's intuitionistic fuzzy relation and complex Atanassov's intuitionistic fuzzy sets. Rosenfeld coined the term "fuzzy subgroups" and established a connection between group theory and fuzzy set theory. As a result, several academics developed fuzzy algebraic structures based on fuzzy sets, intuitionistic fuzzy sets (IFSs), and CIFs (for detail, see [11-18]). Several real and tangible circumstances can be illustrated using a diagram composed of a collection of nodes with lines joining specific pairs of these nodes. The nodes could select individuals, with lines connecting pairs of friends, or primary health care facilities, with lines representing beneficiaries' streets or roads in the region. Fuzzy graph modeling is a useful mathematical tool for solving combinatorial issues in a variety of fields, such as image capturing, computer network, electric network, operations research, social science, road network, topology, optimization, algebra, computer science, environmental science, and scheduling problem. Fuzzy graph theory has an intuitive and aesthetic appeal because of the diagrammatic representation. Due to the natural presence of vagueness and ambiguity, fuzzy graphical models are far superior to graphical models. We needed fuzzy set theory at first to deal with numerous complicated phenomena that had inadequate information. Based on Zadeh's fuzzy connection, Kauffman [19] was the first to coin the term "fuzzy graph." Rosenfeld [20] went on to invent fuzzy vertex, fuzzy edge, and theoretical fuzzy graph ideas like routes, connectedness, and cycle, among other things. Following Mordeson and Chang-Shyh's [21] discussion of fuzzy graph operations, Bhutani and Battou's [22] research of M-strong fuzzy graphs was published. Following that, Eslahchi and Onagh [23], Gani and Malarvizhi [24], Mordeson and Nair [25], and Mathew and Sunitha [26] propose a slew of concepts and definitions, primarily under the headings of vertex strength of fuzzy graphs, fuzzy trees, isomorphism on fuzzy graphs, fuzzy subgraphs, fuzzy paths, and complement of a fuzzy graph. Because the membership function was insufficient to express the complexity of object features, a nonmembership function was created. By combining the nonmembership and hesitation qualities, Atanassov [2] constructed the intuitionistic fuzzy set theory, which was an elaboration of the basic set theory. This idea has been used to a variety of domains, including computer programming, medical fields, decision-making problems, marketing evaluation, and banking issues. In 2006, Parvathi and Karunambigai [27] proposed an intuitionistic fuzzy graph as a variant of Atanassov's IFG. Thirunavukarasu et al. [28] built on this concept by incorporating complex fuzzy graphs. Shannon and Atanassov [29] defined and discussed intuitionistic fuzzy graphs. Later on, a number of authors worked on intuitionistic fuzzy graphs and made several important contributions to the subject (for instance, see [30-33]). Sahoo and Pal discussed different types of products on intuitionistic fuzzy graphs in [34]. Using the concept of a complex intuitionistic fuzzy set, Yaqoob et al. [35] constructed complex intuitionistic fuzzy graphs (CIFGs).

This paper's structure is as follows: the second section dives into some basic definitions. In Section 3, we define the direct product of two CIFGs. We define strong CIFG. We show that the direct product of two CIFGs is a CIFG as well. At the end of this section, we show that if the direct product of two CIFGs is strong, then at least one of them is strong. In Section 4, we define the semidirect product of two CIFGs. This section demonstrates that the semidirect product of two CIFGs is also a CIFG. At the end of this section, we demonstrate that if the semidirect product of two CIFGs is strong, then at least one of them is strong. The strong product of two CIFGs is defined in the fifth section. We demonstrate that the strong direct product of two CIFGs is CIFG. Furthermore, we demonstrated that if the strong product of two CIFGs is strong, then at least one of them is strong. In the last section of this paper, we define the modular product of two CIFGs and examine some intriguing results. We also investigate how the degree of vertex behaves in the modular product of two CIFGs.

\section{Preliminaries}

We go over some basic definitions that will assist us in our future discussions.

Definition 1 [1]. A fuzzy set (FS) $X$ of a nonempty set $A$ is a function, $X: A \longrightarrow[0,1]$.

Definition 2 [2]. An intuitionistic fuzzy set (IFS) $X$ of a universe of discourse $A$ is a triplet of the form $X=\left\{\left\langle a, m_{X}(a)\right.\right.$, $\left.\left.n_{X}(a)\right\rangle \mid a \in A\right\}$, where the functions $m_{X}(a): A \longrightarrow[0,1]$ and $n_{X}(a): A \longrightarrow[0,1]$ are the membership function (degree of truthfulness) and nonmembership functions (degree of falsity), respectively. These functions must fulfill the condition $0 \leq m_{X}(a)+n_{X}(a) \leq 1$.

Definition 3 [36]. The object of the form

$$
X=\left\{\left(a, m_{X}(a) e^{i \alpha_{X}(a)}, n_{X}(a) e^{i \beta_{X}(a)}\right): a \in A\right\},
$$

is a complex intuitionistic fuzzy set (CIFS) defined on universe of discourse $A$. 
Here,

$$
\begin{aligned}
i & =\sqrt{-1}, m_{X}(a), n_{X}(a) \in[0,1], \alpha_{X}(a), \beta_{X}(a) \in[0,2 \pi] \text { and } 0 \\
& \leq m_{X}(a)+n_{X}(a) \leq 1 .
\end{aligned}
$$

Definition 4 [27]. An intuitionistic fuzzy graph is of the form $G=(B, C, X, Y)$ on the crisp graph $G^{*}$ with vertex set $B$ and edge set $C$, where

(1) $B=\left\{b_{1}, b_{2}, \cdots, b_{n}\right\}$ and $X=\left(m_{X}, n_{X}\right)$ such that $m_{X}: B \longrightarrow[0,1]$ and $n_{X}: B \longrightarrow[0,1]$ denote the membership value (MV) and nonmembership value (NMV) of the element $b_{i} \in B$, respectively, such that $m_{X}\left(b_{i}\right)+n_{X}\left(b_{i}\right) \leq 1$ for all $b_{i} \in B$

(2) $C \subseteq B \times B$ and $Y=\left(m_{Y}, n_{Y}\right)$ where $m_{Y}: C \longrightarrow[0,1]$ and $n_{Y}: C \longrightarrow[0,1]$ are defined by $m_{Y}\left(b_{i}, b_{j}\right) \leq m_{X}$ $\left(b_{i}\right) \wedge m_{X}\left(b_{j}\right)$ and $n_{Y}\left(b_{i}, b_{j}\right) \leq n_{X}\left(b_{i}\right) \vee n_{X}\left(b_{j}\right)$ such that $m_{Y}\left(b_{i}, b_{j}\right)+n_{Y}\left(b_{i}, b_{j}\right) \leq 1, \forall\left(b_{i}, b_{j}\right) \in C$

Definition 5 [35]. A complex intuitionistic fuzzy graph (CIFG) with an underlaying vertex set $B$ and edge set $C$ is defined to be a pair $\mathbb{G}=(B, C, X, Y)$, where $X$ is a CIFS on $B$ and $Y$ is a CIFS on $C \subseteq B \times B$ such that

$$
\begin{aligned}
& m_{Y}\left(b_{i}, b_{j}\right) e^{i \alpha_{Y}\left(b_{i}, b_{j}\right)} \leq \min \left\{m_{X}\left(b_{i}\right), m_{X}\left(b_{j}\right)\right\} e^{i \min \left\{\alpha_{X}\left(b_{i}\right), \alpha_{X}\left(b_{j}\right)\right\},} \\
& n_{Y}\left(b_{i}, b_{j}\right) e^{i \beta_{Y}\left(b_{i}, b_{j}\right)} \leq \max \left\{m_{X}\left(b_{i}\right), n_{X}\left(b_{j}\right)\right\} e^{i \max \left\{\beta_{X}\left(b_{i}\right), \beta_{X}\left(b_{j}\right)\right\},}
\end{aligned}
$$

for all $b_{i}, b_{j} \in B$.

Definition 6 [35]. Let $G=(B, C, X, Y)$ be the given CIFG. The degree of a vertex $b_{i}$ in $G$ is defined by

$$
\operatorname{deg}_{G}\left(b_{i}\right)=\left(\sum_{\left(b_{i}, b_{j}\right) \in C} m_{Y}\left(b_{i}, w b_{j}\right) e^{i \alpha_{Y}\left(b_{i} b_{j}\right)}, \sum_{\left(b_{i}, b_{j}\right) \in C} n_{Y}\left(b_{i} b_{j}\right) e^{i \beta_{Y}\left(b_{i} b_{j}\right)}\right) .
$$

\section{Direct Product of Two CIFGs}

Definition 7. The direct product of two CIFGs, $G_{1}=\left(B_{1}\right.$, $\left.C_{1}, X_{1}, Y_{1}\right)$ and $G_{2}=\left(B_{2}, C_{2}, X_{2}, Y_{2}\right)$ such that $B_{1} \cap B_{2}=\phi$ is defined to be CIFG $G_{1} o G_{2}=\left(B, C, X_{1} o X_{2}, Y_{1} o Y_{2}\right)$ where

$$
\begin{aligned}
B & =B_{1} \times B_{2} \text { and } C=C_{1} \times C_{2} \\
& \left.=\left\{\left(b_{i_{1}}, b_{j_{1}}\right),\left(b_{i_{2}}, b_{j_{2}}\right)\right) \mid\left(b_{i_{1}}, b_{i_{2}}\right) \in C_{1},\left(b_{j_{1}}, b_{j_{2}}\right) \in C_{2}\right\} .
\end{aligned}
$$

The MV and NMV for the vertex $\left(b_{i_{1}}, b_{i_{2}}\right)$ in $G_{1} \mathrm{o} G_{2}$ are given by

$$
\begin{aligned}
& \left(m_{X_{1}} e^{i \alpha_{X_{1}}} \mathrm{O} m_{X_{2}} e^{i \alpha_{X_{2}}}\right)\left(b_{i_{1}}, b_{i_{2}}\right)=m_{X_{1}}\left(b_{i_{1}}\right) e^{i \alpha_{X_{1}}\left(b_{i_{1}}\right)} \wedge m_{X_{2}}\left(b_{i_{2}}\right) e^{i \alpha_{X_{2}}\left(b_{i_{2}}\right)} \\
& =\min \left\{m_{X_{1}}\left(b_{i_{1}}\right), m_{X_{2}}\left(b_{i_{2}}\right)\right\} e^{i \min \left\{\alpha_{X_{1}}\left(b_{11}\right), \alpha_{X_{2}}\left(b_{i_{2}}\right)\right\}}, \\
& \left(n_{X_{1}} e^{i \beta_{X_{1}}} 0 n_{X_{2}} e^{i \beta_{X_{2}}}\right)\left(b_{i_{1}}, b_{i_{2}}\right)=n_{X_{1}}\left(b_{i_{1}}\right) e^{i \beta_{X_{1}}\left(b_{i_{1}}\right)} \wedge n_{X_{2}}\left(b_{i_{2}}\right) e^{i \beta_{X_{2}}\left(b_{i_{2}}\right)} \\
& =\max \left\{n_{X_{1}}\left(b_{i_{1}}\right), n_{X_{2}}\left(b_{i_{2}}\right)\right\} e^{i \max \left\{\beta_{X_{1}}\left(b_{11}\right), \beta_{X_{2}}\left(b_{i_{2}}\right)\right\} .}
\end{aligned}
$$

The NM and NMV for the edge $\left(u=\left(b_{i_{1}}, b_{j_{1}}\right), v=\left(b_{i_{2}}\right.\right.$, $\left.b_{j_{2}}\right)$ ) in $G_{1} \Pi G_{2}$ are given by

$$
\begin{aligned}
\left(m_{Y_{1}} e^{i \alpha_{Y_{1}}} \Pi m_{Y_{2}} e^{i \alpha_{Y_{2}}}\right)(u, v) & =m_{Y_{1}}(u) e^{i \alpha m_{Y_{1}}(u)} \wedge m_{Y_{2}}(v) e^{i \alpha m_{Y_{2}}(v)} \\
& =\min \left\{m_{Y_{1}}(u), m_{Y_{2}}(v)\right\} e^{i \min \left\{\alpha_{Y_{1}}(u), \alpha_{Y_{2}}(v)\right\},}
\end{aligned}
$$

$$
\begin{aligned}
\left(n_{Y_{1}} e^{i \beta_{Y_{1}}} \prod n_{Y_{2}} e^{i \beta_{Y_{2}}}\right)(u, v) & =n_{Y_{1}}(u) e^{i \beta_{Y_{1}}(u)} \vee n_{Y_{2}}(v) e^{i \beta_{Y_{2}}(v)} \\
& =\max \left\{n_{Y_{1}}(u), n_{Y_{2}}(v)\right\} e^{i \max \left\{\beta_{Y_{1}}(u), \beta_{Y_{2}}(v)\right.} .
\end{aligned}
$$

Now we define the strong CIFG.

Definition 8. A CIFG $G=(B, C, X, Y)$ is called strong CIFG if

$$
\begin{aligned}
m_{Y}(u, v) e^{i \alpha_{Y}(u, v)} & =\left\{m_{X}(u) e^{i \alpha_{X}(u)} \wedge m_{X}(v) e^{i \alpha_{X}(v)}\right\} \\
& =\min \left\{m_{X}(u), m_{X}(v)\right\} e^{i \min \left\{m_{X}(u), m_{X}(v)\right\}}, \\
n_{Y}(u, v) e^{i \beta_{B}(u, v)} & =\left\{n_{X}(u) e^{i \beta_{X}(u)} \vee n_{X}(v) e^{i \beta_{X}(v)}\right\} \\
& =\max \left\{n_{X}(u), n_{X}(v)\right\} e^{i \max \left\{\beta_{X}(u), \beta_{X}(v)\right\}}
\end{aligned}
$$

Theorem 9. Let $G_{1}=\left(B_{1}, C_{1}, X_{1}, Y_{1}\right)$ and $G_{2}=\left(B_{2}, C_{2}, X_{2}\right.$, $Y_{2}$ ) be two strong CIFGs; then, $G_{1} \Pi G_{2}$ is also a strong CIFG.

Proof. As $G_{1}=\left(B_{1}, C_{1}, X_{1}, Y_{1}\right)$ and $G_{2}=\left(B_{2}, C_{2}, X_{2}, Y_{2}\right)$ are strong CIFGs, so by (9) and (10), we have

$$
\begin{gathered}
\left\{\begin{array}{l}
\left(m_{Y_{1}}\left(u_{1}, v_{1}\right) e^{i \alpha_{Y_{1}}\left(u_{1}, v_{1}\right)}=\min \left\{m_{X_{1}}\left(u_{1}\right), m_{X_{1}}\left(v_{1}\right)\right\} e^{i \min \left\{\alpha_{X_{1}}\left(u_{1}\right), \alpha_{X_{1}}\left(v_{1}\right)\right\},}\right. \\
\left(n_{Y_{1}}\left(u_{1}, v\right) e^{i \beta Y_{1}\left(u_{1}, v_{1}\right)}=\max \left\{n_{X_{1}}\left(u_{1}\right), n_{X_{1}}\left(v_{1}\right)\right\} e^{i \max \left\{\beta_{X_{1}}\left(u_{1}\right), \beta_{X_{1}}\left(v_{1}\right)\right\},}\right.
\end{array}\right. \\
\left\{\begin{array}{l}
\left(m_{Y_{2}}\left(u_{2}, v_{2}\right) e^{i \alpha_{Y_{2}}\left(u_{2}, v_{2}\right)}=\min \left\{m_{X_{2}}\left(u_{2}\right), m_{X_{2}}\left(v_{2}\right)\right\} e^{i \min \left\{\alpha_{X_{2}}\left(u_{2}\right), \alpha_{X_{2}}\left(v_{2}\right)\right\},}\right. \\
\left(n_{Y_{2}}\left(u_{2}, v_{2}\right) e^{i \beta Y_{2}\left(u_{2}, v_{2}\right)}=\max \left\{n_{X_{2}}\left(u_{2}\right), n_{X_{2}}\left(v_{2}\right)\right\} e^{i \max \left\{\beta_{X_{2}}\left(u_{2}\right), \beta_{X_{2}}\left(v_{2}\right)\right\},}\right.
\end{array}\right.
\end{gathered}
$$

for all $\left(u_{1}, v_{1}\right) \in E_{1}$ and $\left(u_{2}, v_{2}\right) \in E_{2}$.

Now from (7) and (8), we have

$$
\begin{aligned}
& \left(m_{Y_{1}} e^{i \alpha Y_{1}} \Pi m_{Y_{2}} e^{i \alpha Y_{2}}\right)\left(\left(b_{i_{1}}, b_{j_{1}}\right),\left(b_{i_{2}}, b_{j_{2}}\right)\right) \\
& =m_{Y_{1}}\left(b_{i_{1}}, b_{i_{1}}\right) e^{i \alpha_{Y_{1}}\left(b_{i_{1}}, b_{j_{1}}\right)} \wedge m_{Y_{2}}\left(b_{j_{2}}, v_{j_{2}}\right) e^{i \alpha_{Y_{2}}\left(b_{i_{2}}, b_{j_{2}}\right)}
\end{aligned}
$$




$$
\begin{aligned}
& =\left[m_{X_{1}}\left(b_{i_{1}}\right) e^{i \alpha X_{1}\left(b_{i_{1}}\right)} \wedge m_{X_{1}}\left(b_{j_{1}}\right) e^{i \alpha X_{1}\left(b_{j_{1}}\right)}\right] \\
& \wedge\left[m_{X_{2}}\left(b_{i_{2}} e^{i \alpha X_{2}} \wedge m_{X_{2}}\left(b_{j_{2}}\right) e^{i \alpha X_{2}\left(b_{j_{2}}\right)}\right]\right. \\
& =\left[m_{X_{1}}\left(b_{i_{1}}\right) e^{i \alpha X_{1}\left(b_{i_{1}}\right)} \wedge m_{X_{2}}\left(b_{j_{2}}\right) e^{i \alpha X_{2}\left(b_{j_{2}}\right)}\right] \\
& \wedge\left[m_{X_{1}}\left(b_{j_{2}}\right) e^{i \alpha X_{1}\left(b_{j_{2}}\right)} \wedge m_{X_{2}}\left(b_{j_{2}}\right) e^{i \alpha X_{2}\left(b_{j_{2}}\right)}\right] \\
& =\left(m_{X_{1}} e^{i \alpha X_{1}} \Pi m_{x_{2}} e^{i \alpha X_{2}}\right)\left(b_{i_{1}}, b_{j_{1}}\right) \\
& \wedge\left(m_{X_{1}} e^{i \alpha X_{1}} \Pi m_{X_{2}} e^{i \alpha X_{2}}\right)\left(b_{i_{2}}, b_{j_{2}}\right) .
\end{aligned}
$$

In addition for nonmembership,

$$
\begin{aligned}
& \left(n_{Y_{1}} e^{i \beta Y_{1}} \mathrm{o} n_{Y_{2}} e^{i \beta Y_{2}}\right)\left(\left(b_{i_{2}}, b_{j_{1}}\right),\left(b_{i_{2}}, b_{j_{2}}\right)\right) \\
& =n_{Y_{1}}\left(b_{i_{1}}, b_{j_{1}}\right) e^{i \beta_{Y_{1}}\left(b_{i_{1}}, b_{j_{1}}\right)} \vee n_{Y_{2}}\left(b_{i_{2}}, b_{j_{2}}\right) e^{i \beta_{Y_{2}}\left(b_{i_{2}}, b_{j_{2}}\right)} \\
& =\left[n_{X_{1}}\left(b_{i_{1}}\right) e^{i \beta X_{1}\left(b_{i_{1}}\right)} \vee n_{X_{1}}\left(b_{i_{2}}\right) e^{i \alpha X_{1}\left(b_{j_{1}}\right)}\right] \\
& \vee\left[n_{X_{2}}\left(b_{i_{2}} e^{i \beta X_{2}} \wedge n_{X_{2}}\left(b_{j_{2}}\right) e^{i \beta X_{2}\left(b_{j_{2}}\right)}\right]\right. \\
& =\left[n_{X_{1}}\left(b_{i_{1}}\right) e^{i \beta X_{1}\left(b_{i_{1}}\right)} \vee n_{X_{2}}\left(b_{j_{2}}\right) e^{i \beta X_{2}\left(b_{j_{2}}\right)}\right] \\
& \vee\left[n_{X_{1}}\left(b_{j_{2}}\right) e^{i \beta X_{1}\left(b_{j_{2}}\right)} \wedge n_{X_{2}}\left(b_{j_{2}}\right) e^{i \beta X_{2}\left(b_{j_{2}}\right)}\right] \\
& =\left(n_{X_{1}} e^{i \beta X_{1}} \Pi n_{X_{2}} e^{i \beta X_{2}}\right)\left(b_{i_{1}}, b_{j_{1}}\right) \\
& \vee\left(n_{X_{1}} e^{i \alpha X_{1}} \Pi n_{X_{2}} e^{i \beta X_{2}}\right)\left(b_{i_{2}}, b_{j_{2}}\right) .
\end{aligned}
$$

This completes the proof.

Theorem 10. Let $G_{1}=\left(B_{1}, C_{1}, X_{1}, Y_{1}\right)$ and $G_{2}=\left(B_{2}, C_{2}, X_{2}\right.$, $Y_{2}$ ) be two CIFGs, such that $G_{1} \Pi G_{2}$ is strong; then, at least one of $G_{1}$ or $G_{2}$ must be strong CIFG.

Proof. Suppose $G_{1}$ and $G_{2}$ are not strong CIFGs. Thus, there exists at least one $\left(b_{i_{1}}, b_{j_{1}}\right) \in B_{1},\left(b_{i_{2}}, b_{j_{2}}\right) \in B_{2}$ such that

$$
\begin{gathered}
m_{Y_{1}}\left(b_{i_{1}}, b_{j_{1}}\right) e^{i \alpha_{Y_{1}}\left(b_{i_{1}}, b_{j_{1}}\right)}<m_{X_{1}}\left(b_{i_{1}}\right) e^{i \alpha_{X_{1}}\left(b_{i_{1}}\right)} \wedge m_{X_{1}}\left(b_{j_{1}}\right) e^{i \alpha_{X_{1}}\left(b_{j_{2}}\right)}, \\
n_{Y_{1}}\left(b_{i_{1}}, b_{j_{1}}\right) e^{i \beta_{Y_{1}}\left(b_{i_{1}}, b_{j_{1}}\right)}<n_{X_{1}}\left(b_{i_{1}}\right) e^{i \beta_{X_{1}}\left(b_{j_{1}}\right)} \vee n_{X_{1}}\left(b_{j_{1}}\right) e^{i \beta_{X_{1}}\left(b_{j_{1}}\right)}, \\
m_{Y_{2}}\left(b_{i_{2}}, b_{j_{2}}\right) e^{i \alpha_{Y_{2}}\left(b_{i_{2}}, b_{j_{2}}\right)}<m_{X_{2}}\left(b_{i_{2}}\right) e^{i \alpha_{X_{2}}\left(b_{i_{2}}\right)} \wedge m_{X_{2}}\left(b_{j_{2}}\right) e^{i \alpha_{X_{2}}\left(b_{j_{2}}\right)}, \\
n_{Y_{2}}\left(b_{i_{2}}, b_{j_{2}}\right) e^{i \beta_{Y_{2}}\left(b_{i_{2}}, b_{j_{2}}\right)}<n_{X_{2}}\left(b_{i_{2}}\right) e^{i \beta_{X_{2}}\left(b_{j_{2}}\right)} \vee n_{X_{2}}\left(b_{j_{2}}\right) e^{i \beta_{X_{2}}\left(b_{j_{2}}\right)}
\end{gathered}
$$

Let $\left(u=\left(b_{i_{1}}, b_{j_{1}}\right), v=\left(b_{i_{2}}, b_{j_{2}}\right)\right) \in B_{1} \times B_{2}$; then,

$$
\begin{aligned}
\left(m_{Y_{1}} e^{i \alpha_{Y_{1}}} \prod m_{Y_{2}} e^{i \alpha_{Y_{2}}}\right)((u, v)) & =m_{Y_{1}}(u) e^{i \alpha_{Y_{1}}(u)} \wedge\left(m_{Y_{2}}(v) e^{i \alpha_{Y_{2}}(v)}\right. \\
& <\left[m_{X_{1}}\left(b_{i_{1}}\right) e^{i \alpha_{X_{1}}\left(b_{i_{1}}\right)} \wedge m_{X_{1}}\left(b_{j_{1}}\right) e^{i \alpha_{X_{1}}\left(b_{j_{1}}\right)}\right] \\
& \wedge\left[m_{X_{2}}\left(b_{i_{2}}\right) e^{i \alpha_{X_{2}}\left(b_{i_{2}}\right)} \wedge m_{X_{2}}\left(b_{j_{2}}\right) e^{i \alpha_{X_{2}}\left(b_{i_{2}}\right)}\right] \\
& =\left[m_{X_{1}}\left(b_{i_{1}}\right) e^{i \alpha_{X_{1}}\left(b_{i_{1}}\right)} \wedge m_{X_{2}}\left(b_{i_{1}}\right) e^{i \alpha_{A_{2}}\left(b_{j_{1}}\right)}\right] \\
& \wedge\left[m_{A_{1}}\left(b_{i_{2}}\right) e^{i \alpha_{A_{1}}\left(b_{i_{1}}\right)} \wedge m_{A_{2}}\left(b_{i_{2}}\right) e^{i \alpha_{X_{2}}\left(b_{i_{2}}\right)}\right] \\
& =\left(m_{X_{1}} e^{i \alpha_{X_{1}}} \Pi m_{X_{2}} e^{i \alpha_{X_{2}}}\right)\left(b_{i_{1}}, b_{j_{1}}\right) \\
& \wedge\left(m_{X_{1}} e^{i \alpha_{X_{1}}} \Pi m_{X_{2}} e^{i \alpha_{X_{2}}}\right)\left(b_{i_{2}}, b_{j_{2}}\right) .
\end{aligned}
$$

Therefore,

$$
\begin{aligned}
& \left(m_{Y_{1}} e^{i \alpha_{Y_{1}}} \Pi m_{Y_{2}} e^{i \alpha_{Y_{2}}}\right)\left(u=\left(b_{i_{1}}, b_{j_{1}}\right), v=\left(b_{i_{2}}, b_{j_{2}}\right)\right)
\end{aligned}
$$

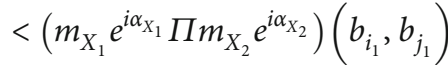

$$
\begin{aligned}
& \wedge\left(m_{X_{1}} e^{i \alpha_{X_{1}}} \Pi m_{X_{2}} e^{i \alpha_{X_{2}}}\right)\left(b_{i_{2}}, b_{j_{2}}\right) \text {. }
\end{aligned}
$$

Again, let $\left(u=\left(b_{i_{1}}, b_{j_{1}}\right), v=\left(b_{i_{2}}, b_{j_{2}}\right)\right) \in B_{1} \times B_{2}$; then,

$$
\begin{aligned}
& \left(n_{Y_{1}} e^{i \beta_{Y_{1}}} \Pi n_{Y_{2}} e^{i \beta_{Y_{2}}}\right)((u, v))=\left(n _ { Y _ { 1 } } ( u ) e ^ { i \beta _ { Y _ { 1 } } ( u ) } \vee \left(n_{Y_{2}}(v) e^{i \beta_{Y_{2}}(v)}\right.\right. \\
& <\left[n_{X_{1}}\left(b_{i_{1}}\right) e^{i \beta_{X_{1}}\left(b_{i_{1}}\right)} \vee n_{X_{1}}\left(b_{j_{1}}\right) e^{i \beta_{X_{1}}\left(b_{j_{1}}\right)}\right] \\
& \vee\left[n_{X_{2}}\left(b_{i_{2}}\right) e^{i \beta_{X_{2}}\left(b_{i_{2}}\right)} \vee n_{X_{2}}\left(b_{j_{2}}\right) e^{i \beta_{X_{2}}\left(b_{i_{2}}\right)}\right] \\
& =\left[n_{X_{1}}\left(b_{i_{1}}\right) e^{i \beta_{X_{1}}\left(b_{i_{1}}\right)} \vee n_{X_{2}}\left(b_{i_{1}}\right) e^{i \beta_{X_{2}}\left(b_{j_{1}}\right)}\right] \\
& \vee\left[n_{X_{1}}\left(b_{i_{2}}\right) e^{i \beta_{X_{1}}\left(b_{i_{1}}\right)} \vee n_{X_{2}}\left(b_{i_{2}}\right) e^{i \beta_{X_{2}}\left(b_{i_{2}}\right)}\right] \\
& =\left(n_{X_{1}} e^{i \beta_{X_{1}}} \Pi n_{X_{2}} e^{i \beta_{X_{2}}}\right)\left(b_{i_{1}}, b_{j_{1}}\right) \\
& \vee\left(n_{X_{1}} e^{i \beta_{X_{1}}} \Pi n_{X_{2}} e^{i \beta_{X_{2}}}\right)\left(b_{i_{2}}, b_{j_{2}}\right) \text {. }
\end{aligned}
$$

Therefore,

$$
\begin{aligned}
& \left(n_{Y_{1}} e^{i \beta_{Y_{1}}} \Pi n_{Y_{2}} e^{i \beta_{Y_{2}}}\right)\left(u=\left(b_{i_{1}}, b_{j_{1}}\right), v=\left(b_{i_{2}}, b_{j_{2}}\right)\right) \\
& <\left(n_{X_{1}} e^{i \beta_{X_{1}}} \Pi n_{X_{2}} e^{i \beta_{X_{2}}}\right)\left(b_{i_{1}}, b_{j_{1}}\right)
\end{aligned}
$$

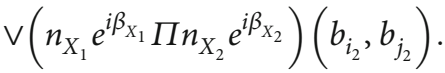

This shows that $G_{1} \Pi G_{2}$ is not strong, which is contradiction.

This completes the proof.

\section{Semistrong Product of Two CIFGs}

Definition 11. The semistrong product of two CIFGs $G_{1}=$ $\left(B_{1}, C_{1}, X_{1}, Y_{1}\right)$ and $G_{2}=\left(B_{2}, C_{2}, X_{2}, Y_{2}\right)$ such that $B_{1} \cap$ $B_{2}=\phi$ is defined to be the CIFG as $G_{1} \downarrow G_{2}=\left(B, C, X_{1}\right.$ 
$\left.X_{2}, Y_{1} \triangleleft Y_{2}\right)$, where $B=B_{1} \times B_{2}$ and $C=\left\{\left(\left(b_{i_{1}}, b_{i_{2}}\right),\left(b_{j_{1}}, b_{j_{2}}\right)\right)\right.$ $\mid\left(b_{i_{1}}, b_{j_{1}}\right) \in B_{1}$ and $b_{i_{2}}=b_{j_{2}}$ or $\left(b_{i_{1}}, b_{j_{1}}\right) \in B_{1}$ and $\left.\left(b_{i_{2}}, b_{j_{2}}\right) \in B_{2}\right\}$.

The MV and NMV of the vertex $(u, v)$ in $G_{1}>G_{2}$ are given as

$$
\begin{aligned}
\left(m_{X_{1}} e^{i \alpha_{X_{1}}}-m_{X_{2}} e^{i \alpha_{X_{2}}}\right)(u, v) & =m_{X_{1}}(u) e^{i \alpha_{X_{1}}(u)} \wedge m_{X_{2}}(v) e^{i \alpha_{X_{2}}(v)} \\
& =\min \left\{m_{X_{1}}(u), m_{X_{2}}(v)\right\} e^{i \min \left\{\alpha_{X_{1}}(u), \alpha_{X_{2}}(v)\right\}} \\
\left(n_{X_{1}} e^{i \beta_{X_{1}}} \checkmark n_{X_{2}} e^{i \beta_{X_{2}}}\right)(u, v) & =n_{X_{1}}(u) e^{i \beta_{X_{1}}(u)} \vee n_{X_{2}}(v) e^{i \beta_{X_{2}}(v)} \\
& =\max \left\{n_{X_{1}}(u), n_{X_{2}}(v)\right\} e^{i \max \left(\beta_{X_{1}}(u), \beta_{X_{2}}(v)\right\}}
\end{aligned}
$$

The MV and NMV for the edge $\left(u, v_{1}\right),\left(u, v_{2}\right)$ and $\left(u_{1}\right.$, $\left.v_{1}\right),\left(u_{2}, v_{2}\right) \in C$ in $G_{1} \triangleleft G_{2}$ are given by as follows:

$$
\begin{gathered}
\left(m_{Y_{1}} e^{i \alpha_{Y_{1}}} m_{Y_{2}} e^{i \alpha_{B_{2}}}\right)\left(\left(u, v_{1}\right),\left(u, v_{2}\right)\right) \\
=m_{X_{1}}(u) e^{i \alpha_{A_{1}}(u)} \wedge m_{Y_{2}}\left(v_{1}, v_{2}\right) e^{i \alpha_{Y_{2}}\left(v_{1}, v_{2}\right)}, \\
\left(m_{Y_{1}} e^{i \alpha_{B_{1}}}{m_{Y_{2}}} e^{i \alpha_{B_{2}}}\right)\left(\left(u_{1}, v_{1}\right),\left(u_{2}, v_{2}\right)\right) \\
=m_{Y_{1}}\left(u_{1}, u_{2}\right) e^{i \alpha_{B_{1}}}(u) \wedge m_{Y_{2}}\left(v_{1}, v_{2}\right) e^{i \alpha_{Y_{2}}\left(v_{1}, v_{2}\right)}, \\
\left(m_{Y_{1}} e^{i \alpha_{B_{1}}}{m_{Y_{2}}}^{i \alpha_{Y_{2}}}\right)\left(\left(u, v_{1}\right),\left(u, v_{2}\right)\right) \\
=m_{X_{1}}(u) e^{i \alpha_{X_{1}}(u)} \wedge m_{Y_{2}}\left(v_{1}, v_{2}\right) e^{i \alpha_{Y_{2}}\left(v_{1}, v_{2}\right)}, \\
\left(m_{Y_{1}} e^{i \alpha_{Y_{1}}} m_{Y_{2}} e^{i \alpha_{Y_{2}}}\right)\left(\left(u_{1}, v_{1}\right),\left(u_{2}, v_{2}\right)\right) \\
=m_{Y_{1}}\left(u_{1}, u_{2}\right) e^{i \alpha_{Y_{1}}(u)} \wedge m_{Y_{2}}\left(v_{1}, v_{2}\right) e^{i \alpha_{Y_{2}}\left(v_{1}, v_{2}\right)} .
\end{gathered}
$$

Theorem 12. If $G_{1}=\left(B_{1}, C_{1}, X_{1}, Y_{1}\right)$ and $G_{2}=\left(B_{2}, C_{2}, X_{2}\right.$, $Y_{2}$ ) are strong CIFGs, then $G_{1} \gg G_{2}$ is also strong.

Proof. If $\left(\left(u, v_{1}\right),\left(u, v_{2}\right)\right) \in C$, then using (19) and (20), we have

$$
\begin{aligned}
& \left(m_{Y_{1}} e^{i \alpha_{Y_{1}}} m_{Y_{2}} e^{i \alpha_{Y_{2}}}\right)\left(\left(u, v_{1}\right),\left(u, v_{2}\right)\right) \\
& \quad=m_{X_{1}}(u) e^{i \alpha_{X_{1}}(u)} \wedge m_{X_{2}}\left(v_{1}, v_{2}\right) e^{i \alpha_{Y_{2}}\left(v_{1}, v_{2}\right)} \\
& \quad=m_{X_{1}}(u) e^{i \alpha_{X_{1}}(u)} \wedge\left(m_{X_{2}}\left(v_{1}\right)^{e_{i \alpha} X_{2}\left(v_{1}\right)} \wedge m_{X_{2}}\left(v_{2}\right)^{e_{i \alpha} X_{2}\left(v_{2}\right)}\right) \\
& \quad=\left(m_{X_{1}}(u) e^{i \alpha_{X_{1}}(u)} \wedge m_{X_{2}}\left(v_{1}\right) e^{i \alpha_{X_{2}}\left(v_{1}\right)}\right) \\
& \quad \wedge\left(m_{X_{2}}(u) e^{i \alpha_{A_{2}}(u)} \wedge m_{X_{2}}\left(v_{2}\right) e^{i \alpha_{A_{2}}\left(v_{2}\right)}\right) \\
& =\left(m_{X_{1}} e^{i \alpha_{X_{1}}}{m_{X_{2}}}^{i \alpha_{X_{2}}}\right)\left(u, v_{1}\right) \\
& \wedge\left(m_{X_{1}} e^{i \alpha_{X_{1}}}{m_{X_{2}}}^{i \alpha_{A_{2}}}\right)\left(u, v_{2}\right) .
\end{aligned}
$$

Similar, we can show that $\left(n_{B_{1}} e^{i \beta B_{1}}-n_{B_{2}} e^{i \beta B_{2}}\right)\left(\left(u, v_{1}\right),(u\right.$, $\left.\left.v_{2}\right)\right)=\left(n_{A_{1}} e^{i \alpha A_{1}} n_{A_{2}} e^{i \alpha A_{2}}\right)\left(u, v_{1}\right) \vee\left(n_{A_{1}} e^{i \alpha A_{1}} n_{A_{2}} e^{i \alpha A_{2}}\right)\left(u, v_{2}\right)$.

Again, if $\left(\left(u_{1}, v_{1}\right),\left(u_{2}, v_{2}\right)\right) \in E$, then using (22) and (24), we have

$$
\begin{aligned}
& \left(m_{Y_{1}} e^{i \alpha_{Y_{1}}}{m_{Y_{2}}}^{i \alpha_{Y_{2}}}\right)\left(\left(u_{1}, v_{1}\right),\left(u_{2}, v_{2}\right)\right. \\
& \quad=m_{Y_{1}}\left(u_{1}, v_{1}\right) e^{i \beta_{Y_{1}}\left(u_{1}, v_{2}\right)} \wedge m_{Y_{2}}\left(u_{2}, v_{2}\right) e^{i \beta_{Y_{2}}\left(u_{2}, v_{2}\right)} \\
& \quad=\left(m_{X_{1}}\left(u_{1}\right) e^{i \alpha_{X_{1}}\left(u_{1}\right)} \wedge m_{X_{2}}\left(v_{1}\right) e^{i \alpha_{X_{2}}\left(v_{1}\right)}\right) \\
& \quad \wedge\left(m_{X_{2}}\left(u_{2}\right) e^{i \alpha_{X_{2}}\left(u_{2}\right)} \wedge m_{X_{2}}\left(v_{2}\right) e^{i \alpha_{X_{2}}\left(v_{2}\right)}\right) \\
& \quad=\left(m_{X_{1}} e^{i \alpha_{X}}{m_{X_{2}}}^{i \alpha_{X_{2}}}\right)\left(u_{1}, v_{1}\right) \\
& \wedge\left(m_{X_{1}} e^{i \alpha_{A_{1}}}{m_{X_{2}}}^{i \alpha X_{2}}\right)\left(u_{2}, v_{2}\right) .
\end{aligned}
$$

Similarly, we can show that

$$
\begin{aligned}
& \left(n_{Y_{1}} e^{i \beta_{Y_{1}}} n_{Y_{2}} e^{i \beta_{Y_{2}}}\right)\left(\left(u_{1}, v_{1}\right),\left(u_{2}, v_{2}\right)\right) \\
& \quad=\left(n_{X_{1}} e^{i \beta_{X_{1}}} n_{X_{2}} e^{i \beta_{X_{2}}}\right)\left(u_{1}, v_{1}\right) \vee\left(n_{X_{1}} e^{i \beta_{X_{1}}} n_{X_{2}} e^{i \beta X_{2}}\right)\left(u_{2}, v_{2}\right)
\end{aligned}
$$

This completes the proof.

Theorem 13. If $G_{1}=\left(B_{1}, C_{1}, X_{1}, Y_{1}\right)$ and $G_{2}=\left(B_{2}, C_{2}, X_{2}\right.$, $\left.Y_{2}\right)$ are two CIFG, such that $G_{1} G_{2}$ is strong, then at least one of $G_{1}$ or $G_{2}$ must be strong.

\section{Strong Product of Two CIFGs}

Definition 14. The strong product of two CIFGs is $G_{1}=$ $\left(B_{1}, C_{1}, X_{1}, Y_{1}\right)$ and $G_{2}=\left(B_{2}, C_{2}, X_{2}, Y_{2}\right)$ such that $B_{1} \cap$ $B_{2}=\phi$ is defined to be CIFGG $\operatorname{CI}_{1} \otimes G_{2}=\left(B, C, X_{1} \otimes X_{2}, Y_{1}\right.$ $\left.\otimes Y_{2}\right)$ where $B=B_{1} \times B_{2}$ and $C=\left\{\left(u, v_{1}\right), u, v_{2}\right) \mid u \in C_{1}$, $\left.\left.\left.\left.\left(v_{1}, v_{2}\right) \in C_{2}\right\} \cup\left\{\left(u_{1}, v\right), u_{2}, v\right) \mid u_{1}\right), u, v_{2}\right) \in C_{1}\right\} \cup\left\{\left(\left(u_{1}, u_{2}\right)\right.\right.$, $\left.\left.\left(v_{1}, v_{2}\right)\right) \mid\left(u_{1}, u_{2}\right) \in C_{1},\left(v_{1}, v_{2}\right) \in C_{2}\right\}$. The MV and NMV for the vertex $(u, v)$ in $G_{1} \otimes G_{2}$ are given by

$$
\begin{aligned}
& \left(m_{X_{1}} e^{i \alpha_{X_{1}}} \otimes m_{X_{2}} e^{i \alpha_{X_{2}}}\right)(u, v)=m_{X_{1}}(u) e^{i \alpha_{X_{1}}(u)} \wedge m_{X_{2}}(v) e^{i \alpha_{A_{2}}(v)}, \\
& \left(n_{X_{1}} e^{i \beta_{X_{1}}} \otimes n_{X_{2}} e^{i \beta_{X_{2}}}\right)(u, v)=n_{X_{1}}(u) e^{i \beta_{X_{1}}(u)} \vee n_{X_{2}}(v) e^{i \beta_{X_{2}}(v)} .
\end{aligned}
$$

The MV and NMV for edges in $G_{1} \otimes G_{2}$ are given by 


$$
\begin{aligned}
& \begin{cases}\left(m_{Y_{1}} e^{i \alpha_{Y_{1}}} \otimes m_{Y_{2}} e^{i \alpha_{Y_{2}}}\right)\left(\left(u, v_{1}\right),\left(u, v_{2}\right)\right)= & m_{X_{1}}(u) e^{i \alpha_{X_{1}}(u)} \wedge m_{Y_{2}}\left(v_{1}, v_{2}\right) e^{i \alpha_{Y_{2}}\left(v_{1}, v_{2}\right)}, \\
\left(m_{Y_{1}} e^{i \alpha_{Y_{1}}} \otimes m_{Y_{2}} e^{i \alpha_{Y_{2}}}\right)\left(\left(u_{1}, v\right),\left(u_{2}, v\right)\right)= & m_{Y_{1}}\left(u_{1}, u_{2}\right) e^{i \alpha_{Y_{1}}\left(\left(u_{1}, u_{2}\right)\right)} \wedge m_{X_{2}}(v) e^{i \alpha_{X_{2}}(v)}, \\
\left(m_{Y_{1}} e^{i \alpha Y_{1}} \otimes m_{Y_{2}} e^{i \alpha_{Y_{2}}}\right)\left(\left(u_{1}, v_{1}\right),\left(u_{2}, v_{2}\right)\right)= & m_{Y_{1}}\left(u_{1}, v_{2}\right) e^{i \alpha_{Y_{1}}\left(\left(u_{1}, v_{2}\right)\right)} \wedge m_{Y_{2}}\left(u_{1}, v_{2}\right) e^{i \alpha_{Y_{2}}\left(u_{1}, v_{2}\right)},\end{cases} \\
& \begin{cases}\left(n_{Y_{1}} e^{i \beta_{Y_{1}}} \otimes n_{Y_{2}} e^{i \beta_{Y_{2}}}\right)\left(\left(u, v_{1}\right),\left(u, v_{2}\right)\right)= & n_{X_{1}}(u) e^{i \beta_{X_{1}}(u)} \vee n_{Y_{2}}\left(v_{1}, v_{2}\right) e^{i \beta_{Y_{2}}\left(v_{1}, v_{2}\right)}, \\
\left(n_{Y_{1}} e^{i \beta_{Y_{1}}} \otimes n_{Y_{2}} e^{i \beta_{Y_{2}}}\right)\left(\left(u_{1}, v\right),\left(u_{2}, v\right)\right)= & n_{Y_{1}}\left(u_{1}, u_{2}\right) e^{i \beta_{Y_{1}}\left(\left(u_{1}, u_{2}\right)\right)} \vee n_{X_{2}}(v) e^{i \beta_{x_{2}}(v)}, \\
\left(n_{Y_{1}} e^{i \beta_{Y_{1}}} \otimes n_{Y_{2}} e^{i \beta_{Y_{2}}}\right)\left(\left(u_{1}, v_{1}\right),\left(u_{2}, v_{2}\right)\right)= & n_{Y_{1}}\left(u_{1}, v_{2}\right) e^{i \beta_{Y_{1}}\left(\left(u_{1}, v_{2}\right)\right)} \vee n_{Y_{2}}\left(u_{1}, v_{2}\right) e^{i \beta_{Y_{2}}\left(u_{1}, v_{2}\right)} .\end{cases}
\end{aligned}
$$

Theorem 15. If $G_{1}=\left(B_{1}, C_{1}, X_{1}, Y_{1}\right)$ and $G_{2}=\left(B_{2}, C_{2}, X_{2}\right.$, $Y_{2}$ ) are strong CIFGs, then $G_{1} \otimes G_{2}$ is also strong.

Theorem 16. If $G_{1}=\left(B_{1}, C_{1}, X_{1}, Y_{1}\right)$ and $G_{2}=\left(B_{2}, C_{2}, X_{2}\right.$, $\left.Y_{2}\right)$ are two CIFGs, such that $G_{1} \otimes G_{2}$ is strong, then at least one of $G_{1}$ or $G_{2}$ must be strong.

Definition 17. A CIFG $G=(B, C, X, Y)$ is said to be complete if

$$
\begin{aligned}
& m_{Y}(u, v) e^{i \alpha_{Y}(u, v)}=m_{X}(u) e^{i \alpha_{X}(u)} \wedge m_{X}(v) e^{i \alpha_{X}(v)} \\
& n_{Y}(u, v) e^{i \beta_{Y}(u, v)}=n_{X}(u) e^{i \alpha_{X}(u)} \wedge n_{X}(v) e^{i \alpha_{X}(v)}
\end{aligned}
$$

for all $u, v \in B$.

Theorem 18. If $G_{1}=\left(B_{1}, C_{1}, X_{1}, Y_{1}\right)$ and $G_{2}=\left(B_{2}, C_{2}, X_{2}\right.$, $Y_{2}$ ) are two CIFGs, then $G_{1} \otimes G_{2}$ is complete.

Proof. As a strong product of CIFGs is CIFG, and every pair of vertices is adjacent. If $\left(\left(u, v_{1}\right),\left(u, v_{2}\right)\right) \in C$, then using (29) and (30), we have

$$
\begin{aligned}
& \left(m_{Y_{1}} e^{i \alpha_{Y_{1}}} \otimes m_{Y_{2}} e^{i \alpha_{Y_{2}}}\right)\left(\left(u, v_{1}\right),\left(u, v_{2}\right)\right) \\
& \quad=m_{X_{1}}(u) e^{\alpha_{X_{1}}(u)} \wedge m_{Y_{2}}\left(v_{1}, v_{2}\right) e^{\alpha_{Y_{2}}\left(v_{1}, v_{2}\right)} \\
& \quad=m_{X_{1}}(u) e^{\alpha_{X_{1}}(u)} \wedge\left(m_{X_{2}}\left(v_{1}\right) e^{i \alpha_{X_{2}}\left(v_{1}\right)} \wedge m_{X_{2}\left(v_{2}\right)} e^{i \alpha_{X_{2}}\left(v_{2}\right)}\right) \\
& \quad=\left(m_{X_{1}}(u) e^{i \alpha_{X_{1}}(u)} \wedge m_{X_{2}}\left(v_{1}\right) e^{i \alpha_{X_{2}}\left(v_{1}\right)}\right) \\
& \quad \wedge\left(m_{X_{1}}(u) e^{i \alpha_{X_{1}}(u)} \wedge m_{X_{2}}\left(v_{2}\right) e^{i \alpha_{X_{2}}\left(v_{2}\right)}\right) \\
& \quad=\left(m_{X_{1}} e^{i \alpha_{X_{1}}} \otimes m_{X_{2}} e^{i \alpha_{X_{2}}}\right)\left(u, v_{1}\right) \\
& \wedge\left(m_{X_{1}} e^{i \alpha_{X_{1}}} \otimes m_{X_{2}} e^{i \alpha_{X_{2}}}\right)\left(u, v_{1}\right) .
\end{aligned}
$$

And by (31), it follows that

$$
\begin{aligned}
& \left(n_{Y_{1}} e^{i \beta_{Y_{1}}} \otimes n_{Y_{2}} e^{i \beta_{Y_{2}}}\right)\left(\left(u . v_{1}\right),\left(u, v_{2}\right)\right) \\
& =n_{X_{1}}(u) e^{\beta_{X_{1}}(u)} \vee n_{Y_{1}}\left(v_{1}, v_{2}\right) e^{\beta_{Y_{2}}\left(v_{1}, v_{2}\right)} \\
& =n_{X_{1}}(u) e^{\beta_{X_{1}}(u)} \vee n_{Y_{1}}\left(v_{1}, v_{2}\right) e^{\beta_{Y_{2}}\left(v_{1}, v_{2}\right)} \\
& =n_{X_{1}}(u) e^{\beta_{X_{1}}(u)} \vee\left[n_{X_{2}}\left(v_{1}\right) e^{i \beta_{X_{2}}\left(v_{1}\right)} \vee n_{X_{2}\left(v_{2}\right)} e^{i \beta_{X_{2}}\left(v_{2}\right)}\right] \\
& =\left(n_{X_{1}}(u) e^{i \beta_{X_{1}}(u)} \vee n_{X_{2}}\left(v_{1}\right) e^{i \beta_{X_{2}}\left(v_{1}\right)}\right) \\
& \vee\left(n_{X_{1}}(u) e^{i \beta_{X_{1}}(u)} \vee n_{X_{2}}\left(v_{2}\right) e^{i \beta_{X_{2}}\left(v_{2}\right)}\right) \\
& =\left(n_{X_{1}} e^{i \beta_{X_{1}}} \otimes n_{X_{2}} e^{i \beta_{X_{2}}}\right)\left(u, v_{1}\right) \\
& \vee\left(n_{X_{1}} e^{i \beta_{X_{1}}} \otimes n_{X_{2}} e^{i \beta_{X_{2}}}\right)\left(u, v_{1}\right)
\end{aligned}
$$

If $\left(\left(u_{1}, v\right),\left(u_{2}, v\right)\right) \in C$, then

$$
\begin{aligned}
& \left(m_{Y_{1}} e^{i \alpha_{Y_{1}}} \otimes m_{Y_{2}} e^{i \alpha_{Y_{2}}}\right)\left(\left(u_{1}, v\right)\left(u_{2}, v\right)\right) \\
& =m_{Y_{1}}\left(u_{1}, v_{2}\right) e^{i \alpha_{Y_{1}}\left(u_{1}, v_{2}\right)} \wedge m_{X_{2}}(v) e^{i \alpha_{X_{2}}(v)} \\
& =\left(m_{X_{1}}\left(u_{1}\right) e^{i \alpha_{X_{1}}\left(u_{1}\right)} \wedge m_{X_{1}}\left(v_{1}\right) e^{i \alpha_{X_{2}}\left(u_{2}\right)}\right) \\
& \wedge\left(m_{X_{2}}(v) e^{i \alpha_{X_{2}}(v)} \wedge m_{X_{2}}(v) e^{i \alpha_{X_{2}}(v)}\right) \\
& =\left(m_{X_{1}} e^{i \alpha_{X_{1}}} \otimes m_{X_{2}} e^{i \alpha_{X_{2}}}\right)\left(u_{1}, v\right) \\
& \wedge\left(m_{X_{1}} e^{i \alpha_{X_{1}}} \otimes m_{X_{2}} e^{i \alpha X_{2}}\right)\left(u_{2}, v\right) .
\end{aligned}
$$

Similarly,

$$
\begin{gathered}
\left(n_{Y_{1}} e^{i \beta_{Y_{1}}} \otimes n_{Y_{2}} e^{i \beta_{Y_{2}}}\right)\left(\left(u_{1}, v\right)\left(u_{2}, v\right)\right)=\left(n_{X_{1}} e^{i \beta_{X_{1}}} \otimes n_{X_{2}} e^{i \beta_{X_{2}}}\right)\left(u_{1}, v\right), \\
\vee\left(n_{X_{1}} e^{i \beta_{X_{1}}} \otimes n_{X_{2}} e^{i \beta_{X_{2}}}\right)\left(u_{2}, v\right) .
\end{gathered}
$$

Again if $\left(\left(u_{1}, v_{1}\right),\left(u_{2}, v_{2}\right)\right) \in C$, then 


$$
\begin{aligned}
& \left(m_{Y_{1}} e^{i \alpha_{Y_{1}}} \otimes m_{Y_{2}} e^{i \alpha_{Y_{2}}}\right)\left(\left(u_{1}, v_{1}\right)\left(u_{2}, v_{2}\right)\right) \\
& \quad=m_{Y_{1}}\left(u_{1}, v_{1}\right) e^{i \alpha_{Y_{1}}\left(u_{1}, v_{1}\right)} \wedge m_{Y_{2}}\left(v_{1}, v_{2}\right) e^{i \alpha_{Y_{2}}\left(u_{2}, v_{2}\right)} \\
& =\left(m_{X_{1}}\left(u_{1}\right) e^{i \alpha_{X_{1}}\left(u_{1}\right)} \wedge m_{X_{1}}\left(v_{2}\right) e^{i \alpha_{X_{2}}\left(v_{2}\right)}\right) \\
& \wedge\left(m_{X_{2}}\left(v_{1}\right) e^{i \alpha_{X_{2}}\left(v_{1}\right)} \wedge m_{X_{2}}\left(v_{2}\right) e^{i \alpha_{X_{2}}\left(v_{2}\right)}\right) \\
& =\left(m_{X_{1}}\left(u_{1}\right) e^{i \alpha_{X_{1}}\left(u_{1}\right)} \wedge m_{X_{2}}\left(u_{1}\right) e^{i \alpha_{X_{2}}\left(v_{1}\right)}\right) \\
& \wedge\left(m_{X_{1}}\left(u_{2}\right) e^{i \alpha_{X_{2}}\left(u_{2}\right)} \wedge m_{X_{2}}\left(v_{2}\right) e^{i \alpha_{X_{2}}\left(v_{2}\right)}\right) \\
& =\left(m_{X_{1}} e^{i \alpha_{X_{1}}} \otimes m_{X_{2}} e^{i \alpha_{X_{2}}}\right)\left(u_{1}, v_{1}\right) \\
& \wedge\left(m_{X_{1}} e^{i \alpha_{X_{1}}} \otimes m_{X_{2}} e^{i \alpha_{X_{2}}}\right)\left(u_{2}, v_{2}\right) .
\end{aligned}
$$

Similarly,

$$
\begin{gathered}
\left(n_{Y_{1}} e^{i \beta_{Y_{1}}} \otimes n_{B_{2}} e^{i \beta_{Y_{2}}}\right)\left(\left(u_{1}, v_{1}\right)\left(u_{2}, v_{2}\right)\right)=\left(n_{X_{1}} e^{i \beta_{X_{1}}} \otimes n_{X_{2}} e^{i \beta_{X_{2}}}\right)\left(u_{1}, v_{1}\right), \\
\vee\left(n_{X_{1}} e^{i \beta_{X_{1}}} \otimes n_{X_{2}} e^{i \beta_{X_{2}}}\right)\left(u_{2}, v_{2}\right) .
\end{gathered}
$$

This completes the proof.

\section{Modular Product of CIFGs}

Definition 19. Let $\mathrm{G}_{1}=\left(B_{1}, C_{1}, X_{1}, Y_{1}\right)$ and $G_{2}=\left(B_{2}, C_{2}\right.$, $X_{2}, Y_{2}$ ) be two CIFGs with underlying vertex sets $B_{1}$ and $B_{2}$ and edge sets $C_{1}$ and $C_{2}$, respectively. Then, modular product of $G_{1}$ and $G_{2}$ is $G_{1} e G_{2}=\left(B_{1} e B_{2}, C_{1} e C_{2}, X_{1} e X_{2}\right.$, $\left.X_{1} e X_{2}\right)$ with underlying vertex set $B_{1} e B_{2}=\left\{\left(x_{1}, y_{1}\right) \mid x_{1} \in\right.$ $\left.B_{1}, y_{1} \in B_{2}\right\}$ and underlying edge set $C_{1} e C_{2}=\left\{\left(x_{1}, y_{1}\right) x_{2}\right.$, $y_{2} \mid\left(x_{1}, x_{2}\right) \in C_{1},\left(y_{1}, y_{2}\right) \in C_{2}$ or $\left.\left(x_{1}, x_{2}\right) \notin C_{1},\left(y_{1}, y_{2}\right) \notin C_{2}\right\}$ with

$$
\begin{aligned}
m_{X_{1}} e^{i \alpha_{X_{1}}} \mathrm{e} m_{X_{2}} e^{i \alpha_{X_{2}}}\left(x_{1}, y_{1}\right) & =m_{X_{1}}\left(x_{1}\right) e^{i \alpha_{X_{1}}}\left(x_{1}\right) \wedge m_{X_{2}}\left(y_{1}\right) e^{i \alpha_{X_{2}}\left(y_{1}\right)} \\
& =\min \left\{m_{X_{1}}\left(x_{1}\right), m_{X_{2}}\left(y_{1}\right)\right\} e^{i \min \left\{\alpha_{X_{1}}\left(x_{1}\right), \alpha_{X_{2}}\left(y_{1}\right)\right\}},
\end{aligned}
$$

$$
\begin{aligned}
\left(n_{X_{1}} e^{i \beta_{X_{1}}} \mathrm{e} n_{X_{2}} e^{i \beta_{X_{2}}}\right)\left(x_{1}, y_{1}\right) & =n_{X_{1}}\left(x_{1}\right) e^{i \beta_{X_{1}}\left(x_{1}\right)} \vee n_{X_{2}}\left(y_{1}\right) e^{i \beta_{X_{2}}\left(y_{1}\right)} \\
& =\max \left\{n_{X_{1}}\left(x_{1}\right), n_{X_{2}}\left(y_{1}\right)\right\} e^{i \max \left\{\beta_{X_{1}}\left(x_{1}\right), \beta_{X_{2}}\left(y_{1}\right)\right\}} .
\end{aligned}
$$

Here, $x_{1} \in B_{1}$ and $y_{1} \in B_{2}$.

The MV and NMV for edges in $G_{1} e G_{2}$ are given by

$$
\begin{aligned}
& m_{Y_{1}} e^{i \alpha_{Y_{1}}} m_{Y_{2}} e^{i \alpha_{Y_{2}}}\left(\left(x_{1}, y_{1}\right),\left(x_{2}, y_{2}\right)\right) \\
& =\left\{\begin{array}{l}
m_{Y_{1}}\left(x_{1}, x_{2}\right) e^{i \alpha_{Y_{1}}\left(x_{1}, x_{2}\right)} \wedge m_{Y_{2}}\left(x_{1}, x_{2}\right) e^{i \alpha_{Y_{2}}\left(x_{1}, x_{2}\right)}, \\
\operatorname{if}\left(x_{1}, x_{2}\right) \in C_{1},\left(y_{1} y_{2}\right) \in C_{2}, \\
m_{X_{1}}\left(x_{1}\right) e^{i \alpha_{X_{1}}\left(x_{1}\right)}\left(x_{1}\right) \wedge m_{X_{1}}\left(x_{2}\right) e^{i \alpha_{X_{1}}\left(x_{2}\right)} \wedge m_{X_{2}}\left(y_{1}\right) e^{i \alpha_{X_{2}}\left(y_{1}\right)} \wedge m_{Y_{2}}\left(y_{2}\right) e^{i \alpha_{Y_{2}}\left(x_{1}\right)}, \\
\operatorname{if}\left(x_{1}, x_{2}\right) \notin C_{1},\left(y_{1}, y_{2}\right) \notin C_{2},
\end{array}\right.
\end{aligned}
$$

$$
\begin{aligned}
& n_{Y_{1}} e^{i \beta_{Y_{1}}} \mathrm{en}_{Y_{2}} e^{i \beta_{Y_{2}}}\left(\left(x_{1}, y_{1}\right),\left(x_{2}, y_{2}\right)\right) \\
& =\left\{\begin{array}{l}
n_{Y_{1}}\left(x_{1}, x_{2}\right) e^{i \beta_{Y_{1}}\left(x_{1}, x_{2}\right)} \vee n_{Y_{2}}\left(x_{1}, x_{2}\right) e^{i \beta_{Y_{2}}\left(x_{1}, x_{2}\right)}, \\
\text { if }\left(x_{1}, x_{2}\right) \in C_{1},\left(y_{1}, y_{2}\right) \in C_{2}, \\
n_{X_{1}}\left(x_{1}\right) e^{i \beta_{X_{1}}\left(x_{1}\right)}\left(x_{1}\right) \vee n_{X_{1}}\left(x_{2}\right) e^{i \beta_{X_{1}}\left(x_{2}\right)} \vee n_{X_{2}}\left(y_{1}\right) e^{i \beta_{X_{2}}\left(y_{1}\right)} \vee n_{Y_{2}}\left(y_{2}\right) e^{i \beta_{Y_{2}}\left(x_{1}\right)}, \\
\text { if }\left(x_{1}, x_{2}\right) \notin C_{1},\left(y_{1}, y_{2}\right) \notin C_{2} .
\end{array}\right.
\end{aligned}
$$

Theorem 20. Let $G_{1}=\left(B_{1}, C_{1}, X_{1}, Y_{1}\right)$ and $G_{2}=\left(B_{2}, C_{2}\right.$, $\left.Y_{2}, Y_{2}\right)$ be the CIFGs; then, modular product $G_{1} \odot G_{2}$ is also a CIFGs.

Proof. Let $G_{1}=\left(B_{1}, C_{1}, X_{1}, Y_{1}\right)$ and $G_{2}=\left(B_{2}, C_{2}, X_{2}, Y_{2}\right)$ be the CIFGs. We have to prove that $G_{1} \odot G_{2}$ is CIFG. By the definition, $X_{1} \odot X_{2}$ is CIFS on $B_{1} \odot B_{2}$ and $Y_{1} \odot Y_{2}$ is CIFS on $C_{1} \odot C_{2}$. From (40) and (41), we have

$$
\begin{aligned}
m_{Y_{1}} & e^{i \alpha_{Y_{1}}} \mathrm{e} m_{Y_{2}} e^{i \alpha_{Y_{2}}}\left(\left(x_{1}, y_{1}\right),\left(x_{2}, y_{2}\right)\right) \\
& =m_{Y_{1}}\left(x_{1}, x_{2}\right) e^{i \alpha_{Y_{1}}\left(x_{1}, x_{2}\right)} \\
& \wedge m_{Y_{2}}\left(x_{1}, x_{2}\right) e^{i \alpha_{Y_{2}}\left(x_{1}, x_{2}\right)} \mathrm{if}\left(x_{1}, x_{2}\right) \in C_{1}, y_{1} y_{2} \in C_{2} \\
& \leq m_{X_{1}}\left(x_{1}\right) e^{i \alpha_{X_{1}}\left(x_{1}\right)}\left(x_{1}\right) \wedge m_{X_{1}}\left(x_{2}\right) e^{i \alpha_{X_{1}}\left(x_{2}\right)} \\
& \wedge m_{X_{2}}\left(y_{1}\right) e^{i \alpha_{X_{2}}\left(y_{1}\right)} \wedge m_{Y_{2}}\left(y_{2}\right) e^{i \alpha_{Y_{2}}\left(x_{1}\right)}
\end{aligned}
$$

Since $G_{1}$ and $G_{2}$ are CIFGs,

$$
\begin{aligned}
& n_{Y_{1}} e^{i \beta_{Y_{1}}} \odot n_{Y_{2}} e^{i \beta_{Y_{2}}}\left(\left(x_{1}, y_{1}\right),\left(x_{2}, y_{2}\right)\right) \\
& =n_{Y_{1}}\left(x_{1}, x_{2}\right) e^{i \beta_{Y_{1}}\left(x_{1}, x_{2}\right)} \vee n_{Y_{2}}\left(x_{1}, x_{2}\right) e^{i \beta_{Y_{2}}\left(x_{1}, x_{2}\right)} \\
& \quad \operatorname{if}\left(x_{1}, x_{2}\right) \in C_{1},\left(y_{1} y_{2}\right) \in C_{2} \\
& \leq n_{X_{1}}\left(x_{1}\right) e^{i \beta_{X_{1}}\left(x_{1}\right)}\left(x_{1}\right) \vee n_{X_{1}}\left(x_{2}\right) e^{i \beta_{X_{1}}\left(x_{2}\right)} \\
& \vee n_{X_{2}}\left(y_{1}\right) e^{i \beta_{X_{2}}\left(y_{1}\right)} \vee n_{Y_{2}}\left(y_{2}\right) e^{i \beta_{Y_{2}}\left(x_{1}\right)} .
\end{aligned}
$$

Since $G_{1}$ and $G_{2}$ are CIFGs,

$$
\begin{aligned}
m_{Y_{1}} & e^{i \alpha_{Y_{1}}} \bigodot m_{Y_{2}} e^{i \alpha_{Y_{2}}}\left(\left(x_{1}, y_{1}\right),\left(x_{2}, y_{2}\right)\right) \\
& =m_{X_{1}}\left(x_{1}\right) e^{i \alpha_{X_{1}}\left(x_{1}\right)}\left(x_{1}\right) \wedge m_{X_{1}}\left(x_{2}\right) e^{i \alpha_{X_{1}}\left(x_{2}\right)} \\
& \wedge m_{X_{2}}\left(y_{1}\right) e^{i \alpha_{X_{2}}\left(y_{1}\right)} \\
& \wedge m_{Y_{2}}\left(y_{2}\right) e^{i \alpha_{Y_{2}}\left(x_{1}\right)} \mathrm{if}\left(x_{1}, x_{2}\right) \notin C_{1},\left(y_{1}, y_{2}\right) \notin C_{2} \\
& =m_{Y_{1}} e^{i \alpha_{Y_{1}}} \bigodot m_{Y_{2}} e^{i \alpha_{Y_{2}}}\left(\left(x_{1}, y_{1}\right),\left(x_{2}, y_{2}\right)\right) .
\end{aligned}
$$

Since $G_{1}$ and $G_{2}$ are CIFGs,

$$
\begin{aligned}
& n_{Y_{1}} e^{i \beta_{Y_{1}}} \bigodot n_{Y_{2}} e^{i \beta_{Y_{2}}}\left(\left(x_{1}, y_{1}\right),\left(x_{2}, y_{2}\right)\right) \\
& \quad=n_{X_{1}}\left(x_{1}\right) e^{i \beta_{X_{1}}\left(x_{1}\right)}\left(x_{1}\right) \vee n_{X_{1}}\left(x_{2}\right) e^{i \beta_{X_{1}}\left(x_{2}\right)} \\
& \quad \vee n_{X_{2}}\left(y_{1}\right) e^{i \beta_{X_{2}}\left(y_{1}\right)} \\
& \quad \vee n_{Y_{2}}\left(y_{2}\right) e^{i \beta_{Y_{2}}\left(x_{1}\right)} \mathrm{if}\left(x_{1}, x_{2}\right) \notin C_{1},\left(y_{1}, y_{2}\right) \notin C_{2} \\
& =n_{Y_{1}} e^{i \beta_{Y_{1}}} \odot n_{Y_{2}} e^{i \beta_{Y_{2}}}\left(\left(x_{1}, y_{1}\right),\left(x_{2}, y_{2}\right)\right) .
\end{aligned}
$$


Hence,

$$
\begin{aligned}
& m_{Y_{1}} e^{i \alpha_{Y_{1}}} \bigodot m_{Y_{2}} e^{i \alpha_{Y_{2}}}\left(\left(x_{1}, y_{1}\right),\left(x_{2}, y_{2}\right)\right) \\
& \quad \leq m_{X_{1}} e^{i \alpha_{X_{1}}} \bigodot m_{X_{2}} e^{i \alpha_{X_{2}}}\left(x_{1}, y_{1}\right) \wedge m_{X_{1}} e^{i \alpha_{X_{1}}} \bigodot m_{X_{2}} e^{i \alpha_{X_{2}}}\left(x_{2}, y_{2}\right), \\
& n_{Y_{1}} e^{i \beta_{Y_{1}}} \bigodot n_{Y_{2}} e^{i \beta_{Y_{2}}}\left(\left(x_{1}, y_{1}\right),\left(x_{2}, y_{2}\right)\right) \\
& \quad \leq n_{X_{1}} e^{i \beta_{X_{1}}} \bigodot m_{X_{2}} e^{i \beta_{X_{2}}}\left(x_{1}, y_{1}\right) \vee n_{X_{1}} e^{i \beta_{X_{1}}} \bigodot n_{X_{2}} e^{i \beta_{X_{2}}}\left(x_{2}, y_{2}\right) .
\end{aligned}
$$

Hence, $G_{1} \mathrm{e} G_{2}$ is CIFG.

Theorem 21. Let $G_{1}=\left(B_{1}, C_{1}, X_{1}, Y_{1}\right)$ and $G_{2}=\left(B_{2}, C_{2}\right.$, $\left.X_{2}, Y_{2}\right)$ be strong CIFGs. Then, modular product $G_{1} \bigodot G_{2}$ is also a strong CIFG.

Theorem 22. Let $G_{1}=\left(B_{1}, C_{1}, X_{1}, Y_{1}\right)$ and $G_{2}=\left(B_{2}, C_{2}\right.$, $\left.X_{2}, Y_{2}\right)$ be two complete CIFGs.
(1) If $m_{Y_{1}}(x) \leq m_{Y_{2}}(x), \alpha_{Y_{1}}(x) \leq \alpha_{Y_{2}}(x)$ and $n_{Y_{1}}(x) \geq$ $n_{Y_{2}}(x), \beta_{Y_{1}}(x) \geq \beta_{Y_{2}}(x)$, then $\operatorname{deg}_{G_{1} e G_{2}}(x, y)=\operatorname{deg}_{G_{1}}$ $(x)$

(2) If $m_{Y_{1}}(x) \geq m_{Y_{2}}(x), \alpha_{Y_{1}}(x) \geq \alpha_{Y_{2}}(x)$ and $n_{Y_{1}}(x) \leq$ $n_{Y_{2}}(x), \beta_{Y_{1}}(x) \leq \beta_{Y_{2}}(x)$, then $\operatorname{deg}_{G_{1} e G_{2}}(x, y)=\operatorname{deg}_{G_{2}}$ $(x)$

Proof.

(1) Let $G_{1}=\left(B_{1}, C_{1}, X_{1}, Y_{1}\right)$ and $G_{2}=\left(B_{2}, C_{2}, X_{2}, Y_{2}\right)$ be two complete CIFGs. The degree of vertex $(x$, $y) \in B_{1} \odot B_{2}$ is $\operatorname{deg}_{G_{1} \odot G_{2}}(x, y)=\left(\operatorname{deg}_{1_{G_{1} \odot G_{1}}}(x, y)\right.$, de $\left.\mathrm{g}_{2_{G_{1} \odot G_{1}}}(x, y)\right)$. From (4), we have

$$
\begin{aligned}
d_{1 G_{1} \odot G_{2}}\left(x_{1}, y_{1}\right)= & \sum_{\left(x_{1} x_{2}\right) \in C_{1},\left(y_{1} y_{2}\right) \in C_{2}}\left(m_{Y_{1}}\left(x_{1}, x_{2}\right) \wedge m_{Y_{2}}\left(y_{1}, y_{2}\right)\right) e^{\alpha_{Y_{1}}\left(x_{1}, x_{2}\right) \wedge m_{Y_{2}}\left(y_{1}, y_{2}\right)} \\
& +\sum_{\left(x_{1} x_{2}\right) \notin C_{1},\left(y_{1} y_{2}\right) \notin C_{2}}\left(m_{X_{1}}\left(x_{1}\right) \wedge m_{X_{1}}\left(x_{2}\right) \wedge m_{X_{2}}\left(y_{1}\right) \wedge m_{X_{2}}\left(y_{1}\right)\right) e^{\alpha_{X_{1}}\left(x_{1}\right) \wedge \alpha_{X_{1}}\left(x_{2}\right) \wedge \alpha_{X_{2}}\left(y_{1}\right) \wedge \alpha_{X_{2}}\left(y_{2}\right)} \\
= & \sum_{\left(x_{1} x_{2}\right) \in C_{1},\left(y_{1} y_{2}\right) \in C_{2}}\left(m_{Y_{1}}\left(x_{1}, x_{2}\right) \wedge m_{Y_{2}}\left(y_{1}, y_{2}\right)\right) e^{\alpha_{Y_{1}}\left(x_{1}, x_{2}\right) \wedge \alpha_{Y_{2}}\left(y_{1}, y_{2}\right)} .
\end{aligned}
$$

Since both $G_{1}$ and $G_{2}$ are complete CIFGs, it follows that

Similarly, $\operatorname{deg}_{1_{G_{1} \odot G_{2}}}\left(x_{1}, y_{1}\right)=\operatorname{deg}_{1_{G_{1} \odot G_{2}}}\left(x_{1}\right)$.

$$
\begin{aligned}
\operatorname{deg}_{1 \mathrm{G}_{1} \odot \mathrm{G}_{2}}\left(x_{1}, y_{1}\right)= & \sum_{\left(x_{1}, x_{2}\right) \in C_{1},\left(y_{1}, y_{2}\right) \in C_{2}}\left(m_{Y_{1}}\left(x_{1}, x_{2}\right) \wedge m_{Y_{2}}\left(y_{1}, y_{2}\right)\right) e^{\alpha_{Y_{1}}\left(x_{1}, x_{2}\right) \wedge m_{Y_{2}}\left(y_{1}, y_{2}\right)} \\
& +\sum_{\left(x_{1}, x_{2}\right) \notin C_{1},\left(y_{1}, y_{2}\right) \notin C_{2}}\left(m_{X_{1}}\left(x_{1}\right) \wedge m_{X_{1}}\left(x_{2}\right) \wedge m_{X_{2}}\left(y_{1}\right) \wedge m_{X_{2}}\left(y_{1}\right)\right) e^{\alpha_{X_{1}}\left(x_{1}\right) \wedge \alpha_{X_{1}}\left(x_{2}\right) \wedge \alpha_{X_{2}}\left(y_{1}\right) \wedge \alpha_{X_{2}}\left(y_{2}\right)} \\
= & \sum_{\left(x_{1}, x_{2}\right) \in C_{1},\left(y_{1}, y_{2}\right) \in C_{2}}\left(m_{Y_{1}}\left(x_{1}, x_{2}\right) \wedge m_{Y_{2}}\left(y_{1}, y_{2}\right)\right) e^{\alpha_{Y_{1}}\left(x_{1}, x_{2}\right) \wedge \alpha_{Y_{2}}\left(y_{1}, y_{2}\right)} .
\end{aligned}
$$

Since both $G_{1}$ and $G_{2}$ are complete CIFGs, it follows that $\operatorname{deg}_{1_{G_{1} \odot G_{2}}}\left(x_{1}, y_{1}\right)=\operatorname{deg}_{1_{G_{1} \odot G_{2}}}\left(x_{1}\right)$.

(2) Omitted

\section{Conclusions}

Graphs are a strong and adaptable data structure for representing real-world connections between different types of data. Graph operations take existing graphs and build new ones. In this investigation, we looked at some interesting results from the key operations direct, semistrong, strong, and modular products for complex intuitionistic fuzzy graphs. A strong complex intuitionistic fuzzy graph is also defined, as well as a number of noteworthy findings. We also look at how a vertex's degree behaves in the modular product of two complex intuitionistic fuzzy graphs.

\section{Data Availability}

No data were used to support this study. 


\section{Conflicts of Interest}

The authors declare no conflicts of interest.

\section{Acknowledgments}

This study was supported by The University of the Lahore, Pakistan.

\section{References}

[1] L. A. Zadeh, "Fuzzy sets," Information and Control, vol. 8, no. 3, pp. 338-353, 1965.

[2] K. Atanassov, "Intuitionistic fuzzy sets," in VII ITKR's Session, V. Sgurev, Ed., Sofia, 1983.

[3] D. Ramot, R. Milo, M. Friedman, and A. Kandel, "Complex fuzzy sets," IEEE Transactions on Fuzzy Systems, vol. 10, pp. 171-186, 2002.

[4] C. Li, T. Wu, and F. T. Chan, "Self-learning complex neurofuzzy system with complex fuzzy sets and its application to adaptive image noise canceling," Neurocomputing, vol. 94, pp. 121-139, 2012.

[5] C. Li and W. Chiang, "Complex fuzzy computing to time series prediction A multi-swarm PSO learning approach," in Asian Conference on Intelligent Information and Database Systems, pp. 242-251, Springer, Berlin, Heidelberg, 2011.

[6] P. Thirunavukarasu, R. Suresh, and P. Thamilmani, "Applications of complex fuzzy sets," JP Journal of Applied Mathematics, vol. 6, no. 1, pp. 5-22, 2013.

[7] M. Xueling, J. Zhan, M. Khan, M. Zeeshan, S. Anis, and A. S. Awan, "Complex fuzzy sets with applications in signals," Computational and Applied Mathematics, vol. 38, no. 4, pp. 1-34, 2019.

[8] L. Chunshien and T. W. Chiang, "Complex neurofuzzy ARIMA forecasting a new approach using complex fuzzy sets," IEEE Transactions on Fuzzy Systems, vol. 21, no. 3, pp. 567584, 2012.

[9] J. Buckley, "Fuzzy complex numbers," Fuzzy Sets and Systems, vol. 33, no. 3, pp. 333-345, 1989.

[10] A. M. Alkouri and A. R. Salleh, "Complex Atanassov's intuitionistic fuzzy relation," In Abstract and Applied Analysis, vol. 2013, pp. 287-382, 2013.

[11] W. J. Liu, "Fuzzy invariant subgroups and fuzzy ideals," Fuzzy Sets and Systems, vol. 8, pp. 133-139, 1982.

[12] M. Gulzar, F. D. D. Alghazzawi, and M. H. Mateen, "A note on complex fuzzy subfield," Indonesian Journal of Electrical Engineering and Computer Science, vol. 21, pp. 1048-1056, 2021.

[13] M. Gulzar, D. Alghazzawi, M. H. Mateen, and N. Kausar, "A certain class of t-intuitionistic fuzzy subgroups," IEEE access, vol. 8, pp. 163260-163268, 2020.

[14] U. M. Swamy and K. L. N. Swamy, "Fuzzy prime ideals of rings," Journal of Mathematical Analysis and Applications, vol. 134, no. 1, pp. 94-103, 1988.

[15] M. Gulzar, M. H. Mateen, D. Alghazzawi, and N. Kausar, "A novel applications of complex intuitionistic fuzzy sets in group theory," IEEE Access, vol. 8, pp. 196075-196085, 2020.

[16] M. Gulzar, M. H. Mateen, Y. M. Chu, D. Alghazzawi, and G. Abbas, "Generalized direct product of complex intuitionistic fuzzy subrings," International Journal of Computational Intelligence Systems, vol. 14, no. 1, pp. 582-593, 2021.
[17] K. Hur, S. Y. Jang, and H. W. Kang, "Intuitionistic fuzzy subgroups and cosets," Honam Mathematical Journal, vol. 26, pp. 17-41, 2004.

[18] A. Rosenfeld, "Fuzzy groups," Journal of Mathematical Analysis and Applications, vol. 35, pp. 512-517, 1971.

[19] A. Kauffman, Introductionà la théorie des sous-ensembles flous à l'usage des ingenieurs, 1973.

[20] A. Rosenfeld, "Fuzzy graphs," in In Fuzzy sets and their applications to cognitive and decision processes, pp. 77-95, Academic press, 1975.

[21] N. J. Mordeson and P. Chang-Shyh, "Operations on fuzzy graphs,” Information Sciences, vol. 79, pp. 159-170, 1994.

[22] K. R. Bhutani and A. Battou, "On M-strong fuzzy graphs," Information Sciences, vol. 155, no. 1-2, pp. 103-109, 2003.

[23] C. Eslahchi and B. N. Onagh, "Vertex-strength of fuzzy graphs," International Journal of Mathematics and Mathematical Sciences, vol. 2006, 9 pages, 2006.

[24] A. N. Gani and J. Malarvizhi, "Isomorphism on fuzzy graphs," International Journal of Computational and Mathematical Sciences, vol. 2, no. 4, pp. 190-196, 2008.

[25] J. N. Mordeson and P. S. Nair, "Fuzzy graphs and fuzzy hypergraphs," Physica, vol. 46, 2012.

[26] S. Mathew and M. S. Sunitha, "Types of arcs in a fuzzy graph," Information Sciences, vol. 179, no. 11, pp. 1760-1768, 2009.

[27] R. Parvathi and M. G. Karunambigai, "Intuitionistic fuzzy graphs," in Computational Intelligence, Theory and applications, pp. 18-20, International Conference in Germany, 2006.

[28] P. Thirunavukarasu, R. Suresh, and K. K. Viswanathan, "Energy of a complex fuzzy graph," Int. J. Math. Sci. Eng. Appl., vol. 10, pp. 243-248, 2016.

[29] A. Shannon and K. T. Atanassov, "A first step to a theory of the intuitionistic fuzzy graphs," in Proceeding of Fuzzy Based Expert System, D. Lakov, Ed., pp. 59-61, Springer, Berlin, 1994.

[30] S. Zeng, M. Shoaib, S. Ali, F. Smarandache, H. Rashmanlou, and F. Mofidnakhaei, "Certain properties of single-valued neutrosophic graph with application in food and agriculture organization," International Journal of Computational Intelligence Systems, vol. 14, no. 1, pp. 1516-1540, 2021.

[31] A. N. Gani and S. S. Begum, "Degree, order and size in intuitionistic fuzzy graphs," International JournalAlgorithm of Computer Mathematics, vol. 3, no. 3, pp. 11-16, 2010.

[32] S. Sahoo and M. Pal, "Intuitionistic fuzzy competition graphs," Journal of Applied Mathematics and Computing, vol. 52, no. 1, pp. 37-57, 2016.

[33] S. Sahoo and M. Pal, "Intuitionistic fuzzy tolerance graphs with application," Journal of Applied Mathematics and Computing, vol. 55, no. 1, pp. 495-511, 2017.

[34] S. Sahoo and M. Pal, "Different types of products on intuitionistic fuzzy graphs," Pacific Science Review A: Natural Science and Engineering, vol. 17, no. 3, pp. 87-96, 2015.

[35] N. Yaqoob, M. Gulistan, S. Kadry, and H. A. Wahab, "Complex intuitionistic fuzzy graphs with application in cellular network provider companies," Mathematics, vol. 7, no. 1, p. 35, 2019.

[36] A. M. Alkouri and A. R. Salleh, "Complex intuitionistic fuzzy sets," AIP Conf. Proc., vol. 1482, pp. 464-470, 2012. 